Article

\title{
Improved Kalman Filter Variants for UAV Tracking with Radar Motion Models
}

\author{
Yuan Wei ${ }^{1}\left(\mathbb{D}\right.$, Tao Hong ${ }^{2,3}$ (D) and Michel Kadoch ${ }^{4, *}$ \\ 1 School of Electronic and Information Engineering, Beihang University, Beijing 100191, China; \\ weiyuan361@buaa.edu.cn \\ 2 Yunnan Innovation Institute·BUAA, Kunming 650233, China; hongtao@buaa.edu.cn \\ 3 Beijing Key Laboratory for Microwave Sensing and Security Applications, Beihang University, \\ Beijing 100191, China \\ 4 Department of Electrical Engineering, ETS, University of Quebec, Montreal, QC H1A 0A1, Canada \\ * Correspondence: michel.kadoch@etsmtl.ca
}

Received: 8 April 2020; Accepted: 30 April 2020; Published: 7 May 2020

\begin{abstract}
Unmanned aerial vehicles (UAV) have made a huge influence on our everyday life with maturity of technology and more extensive applications. Tracking UAVs has become more and more significant because of not only their beneficial location-based service, but also their potential threats. UAVs are low-altitude, slow-speed, and small targets, which makes it possible to track them with mobile radars, such as vehicle radars and UAVs with radars. Kalman filter and its variant algorithms are widely used to extract useful trajectory information from data mixed with noise. Applying those filter algorithms in east-north-up (ENU) coordinates with mobile radars causes filter performance degradation. To improve this, we made a derivation on the motion-model consistency of mobile radar with constant velocity. Then, extending common filter algorithms into earth-centered earth-fixed (ECEF) coordinates to filter out random errors is proposed. The theory analysis and simulation shows that the improved algorithms provide more efficiency and compatibility in mobile radar scenes.
\end{abstract}

Keywords: unmanned aerial vehicle (UAV); Kalman filter; east-north-up (ENU) coordinate; earth-centered earth-fixed (ECEF) coordinate; mobile radar

\section{Introduction}

Unmanned Aerial Vehicles (UAVs), also named drones, have attracted considerable attention because of their widespread use and potential threats. Aerial photography, agriculture, plant protection, express delivery, disaster relief, wildlife observation, infectious-disease monitoring, mapping, news reports, power inspection, disaster management [1], movie and television photography are common UAV applications. However, UAVs have brought many threats such as disturbing regular flight, attacking by carried dangerous goods, spying, border smuggling, and drug trafficking. It is necessary and urgent to detect and track UAVs. Computer vision [2], capturing remote control [3] and radar detection [4] are common methods to identify UAVs. The rapid development and application of internet-of-things (IoT) and fifth-generation (5G) technology prompt the economic value of UAVs across many civil application fields [5]. Many 5G-based detecting and tracking methods are invented [6]. Researchers and engineers will continue paying more attention to identify and track UAVs.

Radar automatic target recognition is a common application in radar systems. Fixed radars are commonly used to detect objects. There are also many mobile types of radars, such as vehicle-borne radars, space-borne radars, airborne radars, ship-borne radars. The carrying capacity of UAV makes it suitable to accomplish detecting mission with onboard radars. Pule Doppler and synthetic-aperture radar (SAR) are common technological regimes of radars on UAVs and radars on UAVs are used to 
monitor, intercept, search, and track ground targets and so on. Authors in [7] and [8] introduced UAV-carried synthetic-aperture radar to detection buried objects. Tracking moving targets with UAV-borne radars is another attractive topic. Authors in [9] discussed identification and classification of ground vehicles by micro-Doppler with radars on UAVs. As a moving target in the air, UAVs can also be detected by UAV-borne radars. However, the work mentioned above focuses on UAV detection and identification, not continuous tracking of UAVs or UAV trajectory acquisition.

The task of continuous tracking and trajectory acquisition is a state estimation problem. Kalman filter is adopted to filter stochastic measurement errors in linear radar systems [10]. However, nonlinear systems are more common in practical applications. For instance, in target tracking applications, the motion models are set up in space rectangular coordinates, while the measurement models are often set up in three-dimensional spherical coordinates [11]. During the data processing, it is necessary to transform coordinates. This is a typical nonlinear problem while the Kalman filter is inappropriate to address this kind of issues. The variants of Kalman filter are developed to address this problem. By amending the Kalman filter model, researchers introduced the extended Kalman filter (EKF) [12], and the unscented Kalman filter (UKF) [13]. These algorithms based on different linearizing techniques attempted to linearize the nonlinear equations and then employ the Kalman filter to accomplish filtering task. Other researchers developed the converted measurement Kalman filter (CMKF) [14] and the unbiased converted measurement Kalman filter (UCMKF) [15].

Kalman filter and its variants are widely used to filter random errors for motion models. A dynamic way to set the process error covariance matrix for a constant-velocity (CV) model Kalman Filter was designed in [16]. Their work featured a marginal computational cost, achieved the best possible final estimated state and reduced the convergence time. They demonstrated their scheme capability by filtering simulated trajectories with low, medium, and high signal-to-noise ratios. Based on their previous study, authors in [17] considered a broader range of constant turn (CT) models and researched UKF as well as EKF variants in terms of their performance and sensitivity to noise parameters. A sequential converted measurement Kalman filter (SCMKF) with Doppler measurements in the ECEF coordinates was introduced in [18]. It worked well for radar installed on a mobile airborne platform with time-varying attitude. The previous works presented lots of tracking objects with mobile radars. We can make use of the same thoughts to track UAVs with mobile radars. Radars on UAVs and vehicles are two of these kinds.

The estimation performance depends on the type of the state coordinates in motion models [17]. In traditional state estimation applications, the filtering is executed in the ENU coordinates, which usually is a local coordinate system [19]. However, filtering in the ENU coordinates will cause filter-performance degradation or even divergence in mobile radars.

In this paper, we attempted to address the filter deviation problem by proposed extended nonlinear filtering algorithms based on ECEF coordinates. We discuss the motion model consistency and figure out the causes of filter performance degradation. Then a concrete process with a constant-velocity model is used to test the validity of our proposed method. We chose MATLAB software to simulate the filter process to testify the performance of the extended algorithms.

The paper is organized as follows. Section 2 introduces the background and a special application scene. Section 3 discusses the motion model consistency and analyzes the reason why filter performance degrades or even diverges. In Section 4, the derivation procedure of extension algorithms in ECEF coordinates is presented. Section 5 gives the simulation results and data analysis. Section 6 is the conclusion of the paper.

\section{Background}

UAVs have a long history. At first, they were invented to perform military missions. Nowadays, they have taken over the consumer market with the development of technology. They play a crucial role in many areas, such as aerial photography, agriculture, plant protection, express delivery, disaster relief, wildlife observation, infectious-disease monitoring, mapping, news reports, power inspection, 
disaster relief, movie and television photography. They have made a big difference to our daily life. However, some negative impact has also been brought in the meantime, such as disturbing the regular flights in no-fly zones, attacks by carrying dangerous objects, and spying on private property and people's privacy. They will be even more dangerous when hijacked by lawbreakers. Border smuggling, drug trafficking, and many other black flight events implemented by UAVs happen a lot and they threaten our normal life and work. Therefore, it becomes urgent and significant to probe, recognize, and track UAVs.

\subsection{When UAV Meets $5 \mathrm{G}$ IoT}

With high data rate, low latency, low power consumption, low-cost, huge system capacity, and massive device connection, fifth-generation mobile networks (5G) bring us a technical revolution and a new communication era. Its huge network capacity and connectivity for billions of devices hugely satisfy the demands of communication in the internet of things (IoT). During the gradual progress from fourth generation (4G) (Long Term Evolution-Advanced (LTE-A) [20], World Interoperability for Microwave Access (WiMAX) [21,22]) to 5G, many technologies have been invented and discussed by researchers and engineers. Authors in [23] proposed an integrated network-function virtualization with software defined radio (SDR) and software defined network (SDN) for 4G/5G networks, and discussed the extension standards and possible extensions for future mobile networks. Ultra-dense heterogeneous networks, self-organizing networks, content-distribution networks, device-to-device communication (D2D), machine-to-machine (M2M) are also key technologies in 5G. With the ever-growing demand for mobile networks, UAV application has been one of the earliest business applications in 5G. UAVs are widely used to provide a reliable and cost-effective solution to wireless communications. By connecting large numbers of base stations with UAVs, a 5G IoT network for future smart-city architecture is proposed in [24]. Their work has an obvious advantage over the existing system by simulation results. Authors in [25] designed a novel link-adaptive constellation-division multiple access (CoDMA) technique by integrating millimeter wave (mmWave) communication with UAV-aided 5G ultra-dense networks. More achievements in the integration of 5G mmWave communications with UAV-assisted wireless networks are surveyed in [26]. The tremendous data processing in 5G demands new technical improvement on cloud computing. Cloud transmission [27] in 5G will make a great difference. As a new computing model, cloud computing can provide secure and reliable data storage and convenient and quick internet service for UAVs. 5G phased arrays in base stations can be used to detect UAVs and monitor automotive traffic, which is discussed in [28]. All these different kinds of applications reveal that UAVs are playing a more and more important role in 5G IoT.

\subsection{Probing, Recognizing and Tracking of $U A V$ s}

\subsubsection{Identify by Computer Vision}

By constituting a node through the deployment of a large number of cameras, and then a visual sensor network will be constituted through many nodes. The UAV's image and information storage will be captured by the whole visual sensor network. The corresponding characteristic information of UAV can be extracted from the captured computer images. After plentiful learning and training, deep learning and neural networks can accomplish recognition and classification of UAVs. But it demands a high-visibility environment. It will never work at night or in severe weather. It also costs too much by deploying huge amounts of cameras.

\subsubsection{Detecting and Interfere UAVs by Capturing Remote Control Signals}

Electromagnetic waves are the only medium of communication and remote control for UAVs. By cutting off the communication link, UAVs can be disturbed. Electromagnetic-pulse bombs and high-power microwave weapons are common tools to interfere UAVs. By means of theoretical analysis, simulation modeling, and physical verification of software radio and the electromagnetic environment, 
researchers did much anti-interference analysis of UAVs' radar echo. But it will not work when the UAVs switch to a silent-flight model.

\subsubsection{Detecting UAVs by Extracting the Micro-Doppler Effect [15]}

Radar target automatic recognition technology plays an important role in radar system. There are some micromotion components on UAVs. The micro-Doppler composition could be extracted from UAVs' radar echo because the rotational motion of micromotion components on UAVs modulates the radar echo. It turns out to be periodic modulation of frequency.

\subsubsection{Identification of UAVs Based on 5G Millimeter-Wave Cellular Infrastructure}

The deployment of ubiquitous cellular networks can also be used to detect black flight UAVs. It is similar to identifying by computer vision, and it is more convenient with the aid of existing cellular base stations, although it may cause greater burdens on base stations. With the abilities of high data rate, low delay, huge system capacity, and massive device connections, 5G will make an enormous influence on our everyday life. Identification of UAVs by $5 \mathrm{G}$ technology will not be a problem, and researchers have done lots of research and exploration.

\subsection{Kalman Filter Algorithm and Its Extended Algorithms}

All filtering algorithms are based on models, which are divided into two categories, linear and nonlinear filtering algorithms. Kalman filter (KF) is one of the typical linear filtering algorithms. Extended Kalman filter (EKF), unscented Kalman filter (UKF), converted measurement Kalman filter (CMKF) and unbiased converted-measurement Kalman filter (UCMKF) are all widely used nonlinear filtering algorithms.

\subsection{A Special Application Scenario}

Figure 1 depicts a typical scene of tracking UAVs There are many types of radars monitoring the airspace, such as UAV radars and vehicle radars. The alien UAV will be discovered when it enters radars' monitoring area. However, not all alien UAVs need to be attacked immediately, some of them just need to be tracked. Subject to the detecting range, the fixed radars are not capable to track UAVs all the time. Therefore, vehicle radars and UAVs with radars will be good choices to accomplish this tracking task.

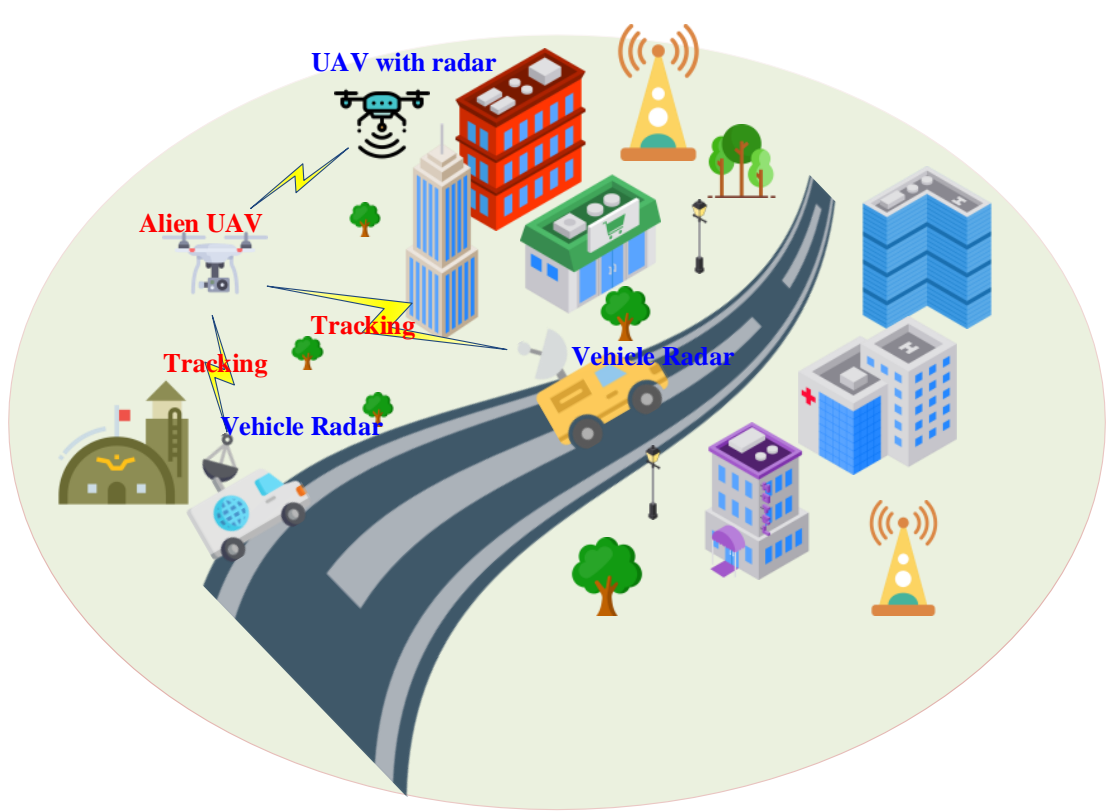

Figure 1. The scene of tracking UAVs with mobile radars. 
In order to obtain the target's locating and tracking information during tracking, we need to carry out data fusion. Measurement noise is inevitable during data acquisition and processing. It is a classical state estimation issue to extract trajectory information. Kalman Filter and its variant algorithms are popular to solve this kind of issue. Meanwhile, a variety of coordinates are employed during data processing. In this paper, we will discuss the filtering performance of EKF, UKF and UCMKF in different coordinates with mobile radars. It suits the above-mentioned scene of tracking UAVs with mobile radars. Finally, we found that filtering in east-north-up (ENU) coordinates is not adequate for scenes with mobile radars. But it works well when accomplishing filtering tasks in earth-centered earth-fixed (ECEF) coordinates, which is possible in the scene shown in Figure 1.

\section{Analysis of Motion Model Consistency during Tracking UAVs}

During tracking objects with radars, relative motion exists. The motion occurs on objects or radars, and maybe both. A motion model is introduced to describe the process. The consistency of the motion model is crucial, because it defines the validity of data processing. Coordinate transformation is an important task to keep the consistency of the motion model.

\subsection{The Applied Coordinates}

Coordinate system is a common auxiliary method of science. In practice, the multiformity of motion models and measuring platforms requires researchers and engineers to adopt many different kinds of coordinates in target tracking applications. Spherical coordinates, east-north-up (ENU) coordinates, earth-centered earth-fixed (ECEF) coordinates, and geodetic coordinate [29] are among widely used coordinates. The motion model consistency is emphasized much during coordinate transformation in detection and tracking. In this paper, we also discuss this issue.

Different coordinates are defined as below.

Spherical coordinates: as part of a 3-D reference system, these consist of range, azimuth, and elevation. Usually, they are used to establish the measurement model [30].

ENU coordinates: these are an approximate inertial coordinate system, and use the barycenter of the platform as the origin. $\mathrm{E}$ is along the tangential of the earth's rotation, $\mathrm{N}$ is along the north, and $\mathrm{U}$ is perpendicular to the plane consisting of the E-axis and $\mathrm{N}$-axis and points up [31]. The ENU coordinates are a local coordinate system where radars locate. Filtering is usually performed in ENU coordinates in conventional-state estimation problems [19].

ECEF coordinates: also known as the earth coordinate system, these are a spatial rectangular coordinate system based on a geographical system. The origin of ECEF coordinates coincides with the earth's center of mass, the x-axis points to the Greenwich meridian, the z-axis points along the Earth's axis, and the $\mathrm{x}$-axis, $\mathrm{y}$-axis and z-axis form right-handed coordinates. Usually, it is used to establish the motion model [30].

Geodetic coordinates: these are built on an ellipsoid surface based on the earth's surface. Latitude, longitude and altitude are the three coordinates. Usually, measured data are from geodetic coordinates. In our study we adopt the World Geodetic System 1984 (WGS-84) coordinates system [32].

In the traditional tracking system, the measurement model is set up in 3-D spherical coordinates and the measured data are from geodetic coordinates. Filtering is performed in ENU coordinates. It works well when radars are motionless or move with a very low velocity. This kind of radar can be applied traditional methods to track UAVs. But our work shows that when moving radars are applied to tracking UAVs, filtering random errors will not be accomplished well by traditional methods. More efficient methods to filter random errors must be developed urgently.

\subsection{Problem of Interest}

In this section, we present a theoretical deduction to show how filtering out random errors in ENU coordinates results in filter performance degradation when tracking UAVs with mobile radars. It disturbs the motion model consistency. 
In practice, the moving states of radars are complicated. The constant-velocity (CV) model, constant-turn (CT) model, and constant-acceleration (CA) model are among common motion models. The real moving state is a set of many single motion models. The CV model is taken as an example to explain motion-model consistency when filtering directly in ENU coordinates.

The state equation and measurement equation are widely used to describe systems:

$$
\left\{\begin{array}{l}
X(k+1)=F X(k)+G w(k) \\
Z(k)=H X(k)+v(k)
\end{array}\right.
$$

where $X(k)$ denotes the state vector at time $k ; F$ denotes the state transition matrix, and usually assumed stationary; $G$ denotes the process noise covariance matrix; $Z(k)$ denotes the actual measurement value of $X$ at time $k ; H$ denotes the measurement matrix; and $w(k)$ and $v(k)$, denote the process noise vector and measurement noise vector, respectively. Here we assume a zero mean and Gaussian white noise. Their covariance matrices are given by:

$$
\left\{\begin{array}{l}
\operatorname{cov}(w(k))=E\left[w(k) w(k)^{T}\right]=Q(k) \\
\operatorname{cov}(v(k))=E\left[v(k) v(k)^{T}\right]=R(k)
\end{array}\right.
$$

In this paper, the constant-velocity (CV) model is employed to discuss the motion-model consistency in the period of coordinate transformation. Providing the radar is fastened on a gyrostabilized platform and all biases are offset, $T$ is the sampling interval of radar, and the motion model state transition matrix will be:

$$
F=\left[\begin{array}{llllll}
1 & T & 0 & 0 & 0 & 0 \\
0 & 1 & 0 & 0 & 0 & 0 \\
0 & 0 & 1 & T & 0 & 0 \\
0 & 0 & 0 & 1 & 0 & 0 \\
0 & 0 & 0 & 0 & 1 & T \\
0 & 0 & 0 & 0 & 0 & 1
\end{array}\right]
$$

Then we can achieve the expression of $G$ and $H$ :

$$
G=\left[\begin{array}{cccccc}
\frac{T}{2} & 1 & 0 & 0 & 0 & 0 \\
0 & 0 & \frac{T}{2} & 1 & 0 & 0 \\
0 & 0 & 0 & 0 & \frac{T}{2}
\end{array}\right]^{T}, H=\left[\begin{array}{llllll}
1 & 0 & 0 & 0 & 0 & 0 \\
0 & 0 & 1 & 0 & 0 & 0 \\
0 & 0 & 0 & 0 & 1 & 0
\end{array}\right]
$$

$(x, \dot{x}, y, \dot{y}, z, \dot{z})^{T}$ describes the components of position and velocity of the state vector $X(k) .(x, y, z)^{T}$ represents position components of measurement vector $Z_{k}$.

Then, we analyze the consistency of the $\mathrm{CV}$ model during the coordinate transformation. Providing that the radar moves with a constant speed with component $\left(V_{x l}(k), V_{y l}(k), V_{z l}(k)\right)$ at time $k$, the location component in geodetic coordinates is $(L(k), \lambda(k), H(k))$ at time $k$.

In the CV model, the velocity component of the target is set as $\left(V_{x 1}, V_{y 1}, V_{z 1}\right)$ in the ECEF coordinates. After transforming to the ENU coordinates, we get the velocity $\left(V_{x}, V_{y}, V_{z}\right)$ :

$$
\left[\begin{array}{c}
V_{x} \\
V_{y} \\
V_{z}
\end{array}\right]=B^{-1}\left\{\left[\begin{array}{c}
V_{x 1} \\
V_{y 1} \\
V_{z 1}
\end{array}\right]-\left[\begin{array}{c}
V_{x l} \\
V_{y l} \\
V_{z l}
\end{array}\right]\right\}
$$


where,

$$
B^{-1}=\left[\begin{array}{ccc}
-\sin \lambda(k) & -\sin L(k) \cos \lambda(k) & \cos L(k) \cos \lambda(k) \\
\cos \lambda(k) & \sin L(k) \sin \lambda(k) & \cos L(k) \sin \lambda(k) \\
0 & \cos L(k) & \sin L(k)
\end{array}\right]^{-1}=\left[\begin{array}{ccc}
b_{11} & b_{12} & b_{13} \\
b_{21} & b_{22} & b_{23} \\
b_{31} & b_{32} & b_{33}
\end{array}\right]
$$

Set:

$$
V_{p}=\left[\begin{array}{c}
V_{x p} \\
V_{y p} \\
V_{z p}
\end{array}\right]=\left[\begin{array}{c}
V_{x 1} \\
V_{y 1} \\
V_{z 1}
\end{array}\right]-\left[\begin{array}{c}
V_{x l} \\
V_{y l} \\
V_{z l}
\end{array}\right]
$$

$V_{p}$ is a constant regardless of random noise, then we get a new expression of $\left(V_{x}, V_{y}, V_{z}\right)$ :

$$
\left\{\begin{array}{l}
V_{x}=b_{11} V_{x p}+b_{12} V_{y p}+b_{13} V_{z p} \\
V_{y}=b_{21} V_{x p}+b_{22} V_{y p}+b_{23} V_{z p} \\
V_{\mathrm{z}}=b_{31} V_{x p}+b_{32} V_{y p}+b_{33} V_{z p}
\end{array}\right.
$$

From Equation (8), we can derive that with motionless radars, there is no difference between the models in ENU coordinates and ECEF coordinates. The velocity component keeps time-invariant despite its changing value. However, when the radar moves, each element in $B^{-1}$ will change, and thereby $\left(V_{x}, V_{y}, V_{z}\right)$ varies, that means the model will no longer be a $\mathrm{CV}$ model while the radar moves. In this situation, the motion model's consistency is aborted. It will cause filter performance degradation during tracking.

The theoretical analysis above shows that the motion model remains unaltered when the radar is motionless. However, when the radar moves, motion models will not remain consistent after the coordinate transformation, the higher velocity, the more drastic changes will happen. The model changes will cause the filtering deviation, and then the system will never accomplish the tracking mission. Now we can conclude that performing the filtering task in ENU coordinates with CV models is not appropriate. We cannot apply ENU coordinates when tracking UAVs with mobile radars.

As Section 2.1 said, in traditional target tracking applications, the motion model is set up in ECEF coordinates and filtering is usually performed in ENU coordinates. But filtering in ENU coordinates is not practicable, which is proved by previous theoretical analysis. Therefore, extending the common filtering algorithms into ECEF coordinates is proposed to solve this issue. Figure 2 shows the flow chart of data transformation among different coordinate systems both in the traditional method and proposed method.

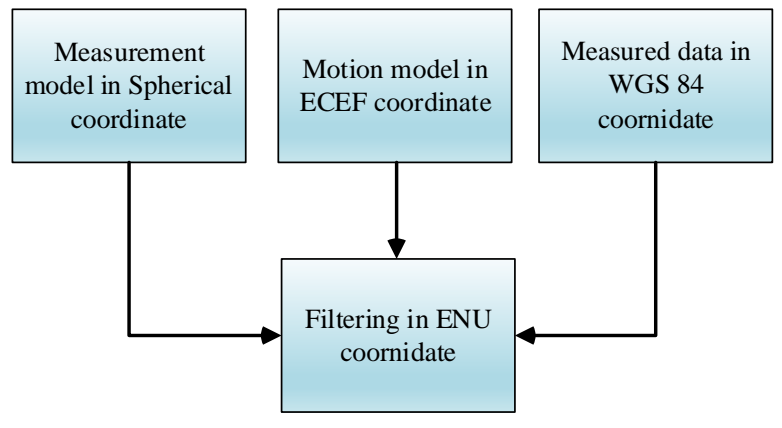

Filtering performance degrades, even diverges

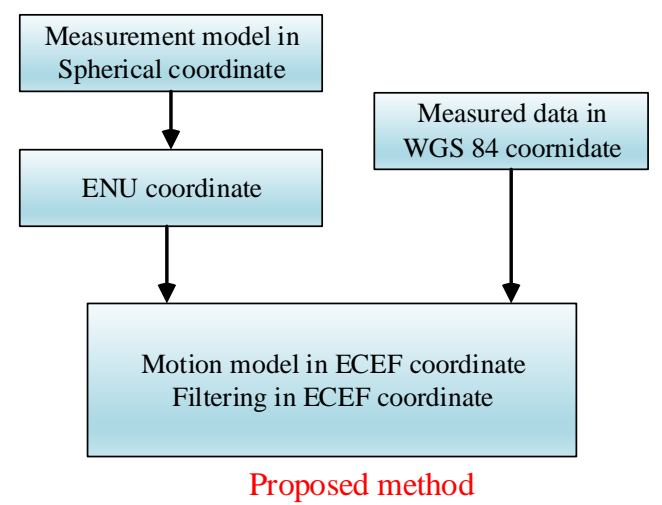

Proposed method

Figure 2. The flow chart of data transformation among different coordinate systems both in the traditional method and proposed method. 


\section{Extended Algorithms Based on ECEF Coordinates}

As mentioned before, filter deviation and even divergence will result from the model inconsistency. This study proposed to carry out the filtering in the ECEF coordinates to ensure the motion model's consistency, and extend common filtering algorithms from the ENU coordinates to the ECEF coordinates.

As mentioned, the measurement data $\widetilde{r}, \widetilde{\theta}, \widetilde{\eta})$ are achieved from the spherical coordinates. $(r, \theta, \eta)$ are the practical measurement data, $\left(r_{\sigma}, \theta_{\sigma}, \eta_{\sigma}\right)$ are the random measurement errors, and $\left(\sigma_{r}, \sigma_{\theta}, \sigma_{\eta}\right)$ are the standard deviation of measurement, which features a zero-mean Gaussian distribution. Registration is applied to eliminate the system errors. $(L(k), \lambda(k), H(k))$ and $\left(x_{s}(k), y_{s}(k), z_{s}(k)\right)$ are position components of the radar in the geodetic coordinates and ECEF coordinates, respectively.

\subsection{EKF Algorithm Based on ECEF Coordinates}

When filtering is performed in the ENU coordinates, the state equations are set in the space rectangular coordinates (namely the ENU coordinates). The radar is located in the ENU coordinates, but the measurement equations are set in the spherical coordinates. The state equation is a linear equation and it is the same as the state equation in (1). The measurement equation is a nonlinear equation. It is expressed as follows:

$$
Z(k)=h[X(k)]+v(k)
$$

The form of $h[X(k)]$ is (A1) showed in Appendix A. It is different from linear systems, the state transition matrix and measurement matrix in nonlinear systems cannot be expressed directly in the form of matrix, but in the form of $f[X(k)]$ and $h[X(k)]$. In order to linearize nonlinear functions, EKF use Taylor expansion in the neighborhood of mean value of the state variable. The measurement matrix $H$ can be achieved by calculating the Jacobian matrix of $h[X(k)]$, as shown in (A2) in Appendix A, where $X(k \mid k-1)$ means the state vector at time $k$, which is predicted from time $k-1 . r=\sqrt{x^{2}+y^{2}+z^{2}}, r_{1}=\sqrt{x^{2}+y^{2}}$.

After calculating $H$, the format is similar to the measurement matrix in the linear system, and the filtering task can be accomplished by Kalman filter.

When applying and extending the application of the EKF from the ENU coordinate to the ECEF coordinate, the calculation of the Jacobian matrix is too complicated to be employed in an engineering application. As the state equation is set in the spherical coordinates, the measurement data have to be converted from ECEF to spherical coordinates. This calculation is too large to consider in this paper. We just show the performance degradation when applying EKF directly in ENU coordinates.

\subsection{UKF Algorithm Based on ECEF Coordinates}

EKF linearizes the nonlinear equations by the first-order Taylor expansion, and then adopts the Kalman filter to accomplish the filtering task. However, the divergence of the approximation and huge calculating burden of the Jacobian matrix will make EKF insignificant in some applications. Therefore, an unscented conversion based on the unscented Kalman filter algorithm was proposed in [31].

UKF calculates the assigned $\sigma$ sample point through an unscented conversion method to linearize the nonlinear equation. The measurement sampling and weight of the $\sigma$ sampling point determine the final measurement prediction. The heavily computation of the Jacobian matrix is avoided by this procedure. In Reference [31], the UKF algorithm based on the ENU coordinates was discussed.

After being calculated from the ECEF coordinate, the state equation will be set up in the geocentric coordinates. To obtain the measurement vector's $\sigma$ sampling, we calculate the sampling point in the ECEF coordinates first and then convert it into the ENU coordinates system. At last, the sampling of a one-step prediction will be determined. The following three steps describe the conversion process: 
1. Calculating the $\sigma$ sampling of the measurement vector:

$$
\left\{\begin{array}{l}
\xi_{k}^{0}=X_{k \mid k-1} \\
\xi_{k}^{i}=X_{k \mid k-1}+\left(\sqrt{(n+\lambda) P_{k \mid k-1}}\right)_{i}, i=1, \ldots, n \\
\xi_{k}^{i}=X_{k \mid k-1}-\left(\sqrt{(n+\lambda) P_{k \mid k-1}}\right)_{i}, i=n+1, \ldots, 2 n
\end{array}\right.
$$

here, $n$ means the dimension of state $X_{k}, n$ equals 6 , and $\lambda$ determines the dispersive degree of the sampling point. It is usually set as a small positive value (for example, 0.01 ). $k$ is usually set as 0 . $\left(\sqrt{(n+\lambda) P_{k \mid k-1}}\right)_{i}$ denotes line $i$ of the square-rooting matrix of matrix $(n+\lambda) P_{k \mid k-1}$.

This step can be divided in four steps, which are shown in Equations (A3)-(A7) in Appendix A.

2. Converting the sample point into the ENU coordinate:

$$
\widetilde{\xi}^{i}(k)=B^{-1}\left\{\left[\begin{array}{c}
\widetilde{\xi}^{i}(k)(1) \\
\widetilde{\xi}^{i}(k)(3) \\
\widetilde{\xi}^{i}(k)(5)
\end{array}\right]-\left[\begin{array}{c}
x_{s}(k) \\
y_{s}(k) \\
z_{s}(k)
\end{array}\right]\right\}
$$

$\widetilde{\xi}^{i}(k)(1), \widetilde{\xi}^{i}(k)(3), \widetilde{\xi}^{i}(k)(5)$ denote the first, third, and fifth components of $\widetilde{\xi}^{i}(k)$.

3. Calculating the measurement prediction:

$$
\begin{gathered}
\zeta^{i}(k \mid k-1)=h\left[\widetilde{\xi}^{i}(k)\right], i=0,1, \ldots, 2 n \\
Z(k \mid k-1)=\sum_{i=0}^{2 n} \omega_{i}^{m} \zeta^{i}(k \mid k-1)
\end{gathered}
$$

After conversion, the filtering task will be accomplished by the common UKF.

\subsection{UCMKF Algorithm Based on ECEF Coordinates}

First of all, we need to convert from the spherical coordinates to the ENU coordinates:

$$
\begin{aligned}
& x=\left(r+r_{\sigma}\right) \cos \left(\eta+\eta_{\sigma}\right) \cos \left(\theta+\theta_{\sigma}\right) \\
& y=\left(r+r_{\sigma}\right) \cos \left(\eta+\eta_{\sigma}\right) \sin \left(\theta+\theta_{\sigma}\right) \\
& z=\left(r+r_{\sigma}\right) \sin \left(\eta+\eta_{\sigma}\right)
\end{aligned}
$$

In the course of above data conversion, the coupling of each component caused by random measurement error will weaken system performance. It is urgent to remove the coupling bias. The authors of [33] developed an unbiased converted-measurement Kalman filter (UCMKF) algorithm in 3-D space to amend the conversion measurements. The corrected measurements $\left(x^{u}, y^{u}, z^{u}\right)$ and covariance matrix $R^{u}$ are given:

$$
\begin{aligned}
& x^{u}=\widetilde{r} \cos \tilde{\eta} \cos \widetilde{\theta}\left(1+\lambda_{\theta} \lambda_{\eta}-\lambda_{\theta}^{-1} \lambda_{\eta}^{-1}\right) \\
& y^{u}=\widetilde{r} \cos \tilde{\eta} \sin \widetilde{\theta}\left(1+\lambda_{\theta} \lambda_{\eta}-\lambda_{\theta}^{-1} \lambda_{\eta}^{-1}\right) \\
& z^{u}=\widetilde{r} \sin \widetilde{\eta}\left(1+\lambda_{\eta}-\lambda_{\eta}^{-1}\right) \\
& x^{u}=\widetilde{r} \cos \widetilde{\eta} \cos \widetilde{\theta}\left(1+\lambda_{\theta} \lambda_{\eta}-\lambda_{\theta}^{-1} \lambda_{\eta}^{-1}\right) \\
& y^{u}=\widetilde{r} \cos \tilde{\eta} \sin \widetilde{\theta}\left(1+\lambda_{\theta} \lambda_{\eta}-\lambda_{\theta}^{-1} \lambda_{\eta}^{-1}\right) \\
& z^{u}=\widetilde{r} \sin \widetilde{\eta}\left(1+\lambda_{\eta}-\lambda_{\eta}^{-1}\right)
\end{aligned}
$$

The elements of matrix $R^{u}$ are given by (A8) in Appendix A. 
$\left(x^{u}, y^{u}, z^{u}\right)$ is the target's corrected position in the ENU coordinates. We transform measurement data into the ECEF coordinates to guarantee the motion model consistency. The target's position $\left(x_{t}, y_{t}, z_{t}\right)$ in the ECEF coordinates will be:

$$
\left[\begin{array}{l}
x_{t} \\
y_{t} \\
z_{t}
\end{array}\right]=\left[\begin{array}{c}
x_{s}(k) \\
y_{s}(k) \\
z_{s}(k)
\end{array}\right]+B_{p}\left[\begin{array}{c}
x^{u} \\
y^{u} \\
z^{u}
\end{array}\right]
$$

where

$$
B=\left[\begin{array}{ccc}
-\sin \lambda(k) & -\sin L(k) \cos \lambda(k) & \cos L(k) \cos \lambda(k) \\
\cos \lambda(k) & \sin L(k) \sin \lambda(k) & \cos L(k) \sin \lambda(k) \\
0 & \cos L(k) & \sin L(k)
\end{array}\right]
$$

The measurement covariance matrix $R_{p}$ in the ECEF coordinates is achieved according to properties of covariance:

$$
R_{p}=B R^{u} B^{T}
$$

Converting the data measurement and the covariance matrix into the ECEF coordinate ensures the motion model's consistency. Therefore, the standard Kalman filter can be applied to filter out the measured data after the conversion; Reference [10] provides the related formula.

\section{Simulation Experiment and Data Analysis}

MATLAB simulation software is a commercial mathematics software with excellent performance in developing algorithms, analyzing data, modeling, and simulating. It was chosen to testify the validity of our proposed algorithms in this study. As mentioned, the calculation of the Jacobian matrix is too complicated to apply the EKF algorithm in the ECEF coordinate, and we only simulate the EKF algorithm in the ENU coordinates. In addition, we also simulate UKF and UCMKF in the ENU coordinate and ECEF coordinate to compare their filtering performance.

\subsection{Experiment Setup}

In reality, we track UAVs with radars installed on vehicles or UAVs. For ease of expression, in our simulation, moving with a CV model, the radar tracks a flying UAV in the sky. Its initial geographical coordinates are $\left[\mathrm{N} 39.9^{\circ}, \mathrm{E} 116.4^{\circ}, 100 \mathrm{~m}\right]$, and geographical coordinates at time $k$ are given by:

$$
\begin{aligned}
& L(k)=L(k-1)+0.001 v \\
& \lambda(k)=\lambda(k) \\
& H(k)=H(k)
\end{aligned}
$$

We choose velocity coefficient $v$ to simulate the real velocity of the moving platform. Here $v$ equals $0,1,10$, and 20, which means the platform is static, moving with low velocity and two higher velocities. We set the radar's sampling interval as 1s, and the standard deviations of the random measurement noises as $\left[100 \mathrm{~m}, 0.08^{\circ}, 0.08^{\circ}\right]$. Registration is already implemented to remove the offset and attitude biases. The initial target's coordinates are [-2921 km, $4726 \mathrm{~km}, 3120 \mathrm{~km}]$ in ECEF coordinates.

The Monte Carlo method is one numerical computation method based on probability and statistics theory, and it can obtain numerical results through large numbers of random repeated sampling.

In this study, the simulation step is set as 50, and the Monte Carlo run number as 100 . The root-mean-square error (RMSE) at time $k$ is:

$$
\operatorname{RMSE}(k)=\sqrt{\frac{1}{N} \sum_{i=1}^{N} M(k)}
$$


Root-mean-square error of position:

$$
M(k)=[\widetilde{x}(k)-x(k)]^{2}+[\widetilde{y}(k)-y(k)]^{2}+[\widetilde{z}(k)-z(k)]^{2}
$$

$x(k), y(k), z(k)$ and $\widetilde{x}(k), \widetilde{y}(k), \widetilde{z}(k)$ denote the real position components and the position components after filtering along $x, y, z$-axis at time $k . N$ is the Monte Carlo simulation number. We evaluate the filter capacity by the root-mean-square error (RMSE), the smaller it is, the better filter capacity.

\subsection{Simulation Results and Discussion}

The results of simulation are presented in Figures 3-6. The black lines with rhombuses depict the raw data's location RMSEs. The green lines with dots show the filtered data's location RMSEs in the ECEF coordinates. The blue lines with triangles describe the filtered data's location RMSEs in the ENU coordinates. The detailed quantized data are displayed in Table 1.
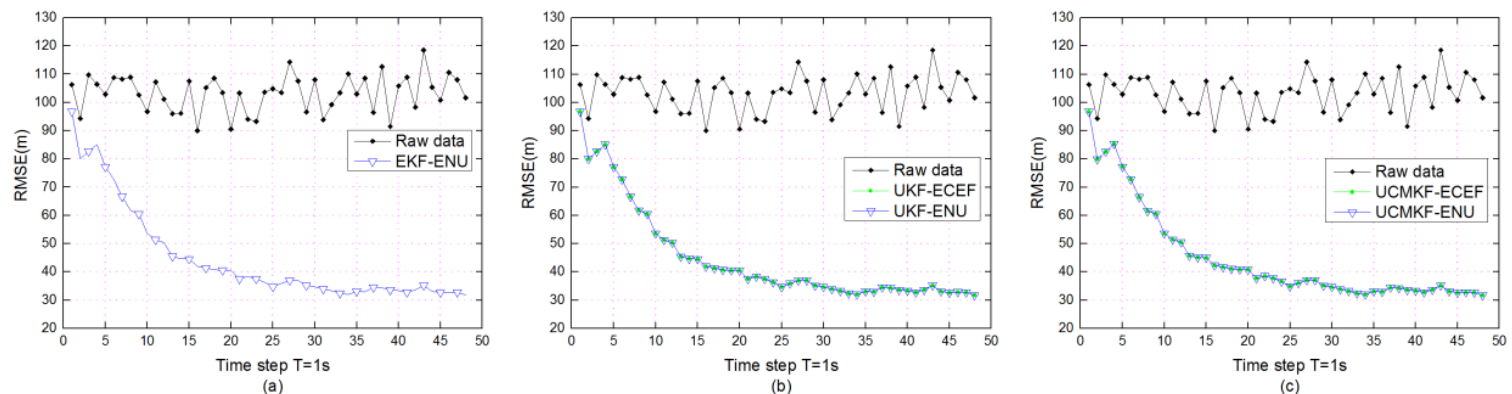

Figure 3. The location RMSE when $v$ is 0: (a) filtering results by EKF based on ENU coordinates; (b) filtering results by UKF based on ENU and ECEF coordinates; (c) filtering results by UCMKF based on ENU and ECEF coordinates.
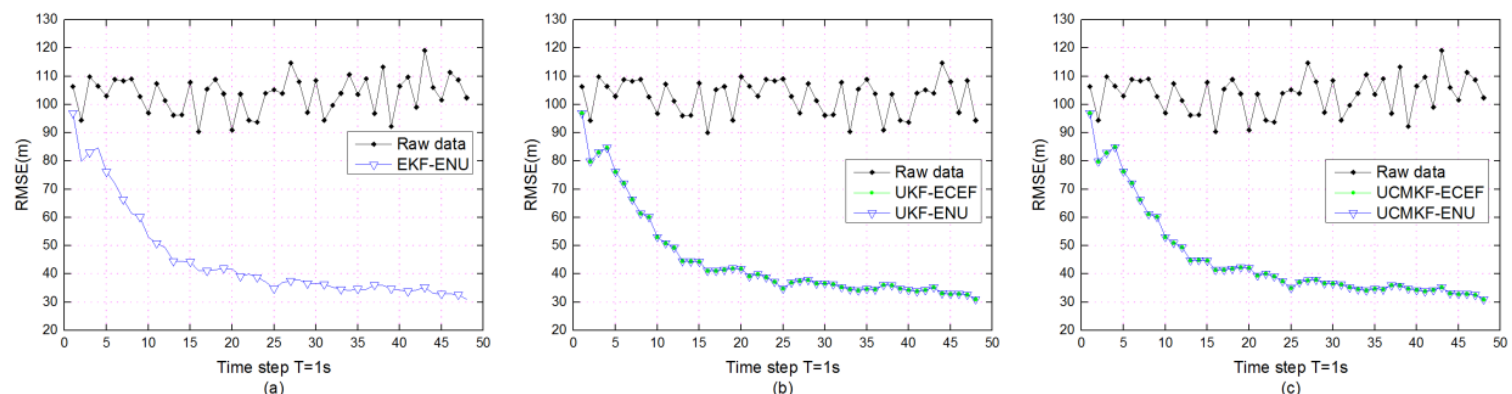

Figure 4. The location RMSE when is 1: (a) filtering results by EKF based on ENU coordinates; (b) filtering results by UKF based on ENU and ECEF coordinates; (c) filtering results by UCMKF based on ENU and ECEF coordinates
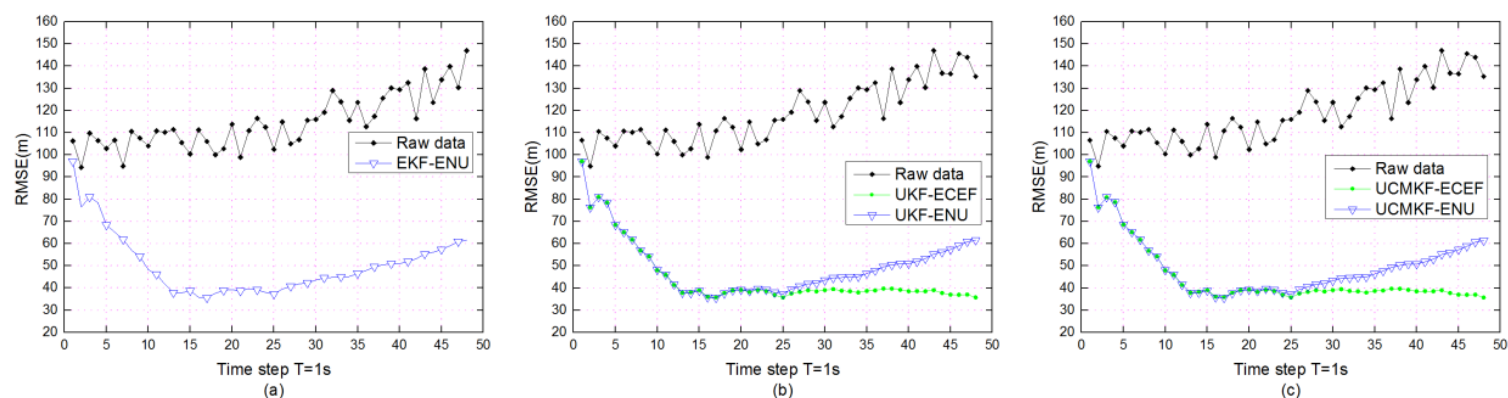

Figure 5. The location RMSE when $v$ is 10: (a) Filtering results by EKF based on ENU coordinates; (b) Filtering results by UKF based on ENU and ECEF coordinates; (c) Filtering results by UCMKF based on ENU and ECEF coordinates. 

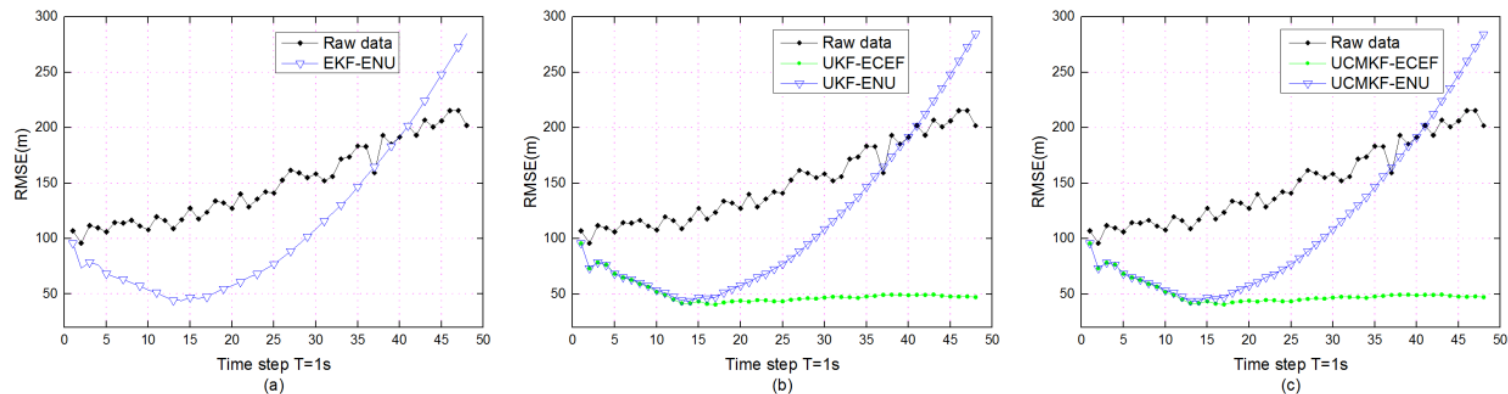

Figure 6. The location RMSE when $v$ is 20: (a) filtering results by EKF based on ENU coordinates; (b) filtering results by UKF based on ENU and ECEF coordinates; (c) filtering results by UCMKF based on ENU and ECEF coordinates.

Table 1. The comparison between root-mean-square errors (RMSEs) of locations by different filter algorithms.

\begin{tabular}{ccccccc}
\hline $\boldsymbol{v}$ & Raw Data & EKF-ENU & UKF-ENU & UKF-ECEF & UCMKF-ENU & UCMKF-ECEF \\
\hline 0 & 103.271 & 44.598 & 44.597 & 44.597 & 44.691 & 44.691 \\
1 & 103.663 & 45.142 & 45.142 & 45.144 & 45.228 & 45.230 \\
10 & 118.379 & 50.176 & 50.181 & 44.752 & 50.085 & 44.770 \\
20 & 149.673 & 113.863 & 113.880 & 50.803 & 113.751 & 50.804 \\
\hline
\end{tabular}

Figures 3-6 show the filtering performance with the different velocity coefficient of radars. The velocity coefficient is 0 in Figure 3, i.e., the radar is static. It is with the situation to tracking UAVs with fixed radars. The filterings by EKF, UKF, and UCMKF all work well enough in the ENU coordinates and these are almost the same as those in ECEF coordinates, as shown in Table 1.

Figure 4 depicts the simulation results when the motion coefficient is set as 1 , which means the radar is moving slowly. Here, the filtering results by EKF, UKF, and UCMKF show no obvious deviations in the ENU coordinate, which is shown in Figure $4 b$,c. The filtering results in ECEF coordinates show no significant differences from those in ENU coordinates. However, the data analysis in Table 1 shows that the filtering accuracy degrades slightly, when filtering is performed in the ECEF coordinates. This is because some errors arise during the coordinate system conversion. In these cases, the traditional method works well and there is no need to worry about the filtering performance degradation.

Figures 5 and 6 show the filtering results in the case of moving radars with high speed. It corresponds to the special situation mentioned in Section 2. As described in Figure 5, when the motion coefficient is 10, the filtering performance in the ENU coordinates degrades. However, Figure $5 b, c$ show that the UKF and UCMKF algorithms in the ECEF coordinates work as well as usual. When the motion coefficient goes up to 20-in other words, the radar moves more quickly-the filtering divergence occurs via the EKF, UKF, and UCMKF algorithms in the ENU coordinates. The data are no longer efficient because their accuracy is lower than the raw data, as shown in Figure 6. This performance degradation will never be accepted. However, filtering in the ECEF coordinates still works well. The proposed method is efficient when tracking UAVs with moving radars.

We can draw a conclusion that in a low-speed CV model (targets and radars move at a constant velocity) the filtering results in both the ENU and ECEF coordinates are efficient. These two methods are both capable for tracking UAVs in this case. When the radar moves quickly enough the filtering degradation and result divergence will occur in the ENU coordinates. Tracking UAVs will fail by this means. We need to filter data in the ECEF coordinates, because that provides more precise filter results no matter how fast the radar moves. The simulation results fit well with the previous theoretical analysis. The proposed extension algorithms have been proved effective by simulation. 


\section{Computational Complexity and Implementation Cost of Proposed Extended Algorithms}

In this section, we will discuss the quality of the proposed extended algorithm. The validity has been approved by simulation results. The advantages of proposed extended algorithms are necessary to investigate.

\subsection{Computational Complexity}

The time complexity of EKF is $O\left(n^{2}\right)$ when process noise and measurement noise feature Gaussian distribution. As mentioned before, the calculation of the Jacobian matrix is too complicated to be employed in an engineering application. We will not consider that in this paper. However, we calculate the time complexity after checking our code. The extended UKF algorithm and UCMKF algorithms are $O\left(n^{3}\right)$ and $O(n)$. The time complexity of UKF is high, but the amount of code is little, so the run time is not long.

In our paper, we chose MATLAB software to simulate the whole calculation of proposed extended algorithms. The simulation ran on a personal laptop. The detailed hardware configurations are Intel Core i3-2310M, 2.1 GHz, 4 GB RAM, and a 120 GB SSD. The total running times on this computer for three algorithms are $2 \mathrm{~s}, 5.2 \mathrm{~s}$, and $2.2 \mathrm{~s}$. The simulation is performed on a laptop, whose performance is not strong enough. When performing this simulation on a desk computer with a more powerful configuration, the run time will be extremely reduced. When applying our extended algorithms on UAVs with radars and vehicle radars, it will cause little burden for radars. It is a lightweight task.

\subsection{Implementation Cost}

In order to track UAVs with mobile radars, we proposed extended filtering algorithms with short running time. Vehicle radars are common after a long development history. The existing vehicle radars can be easily equipped with our algorithms to improve UAV-tracking performance while moving. The implementation cost is not high at all. As for UAVs with radars, Pule Doppler and synthetic-aperture radar (SAR) are common technological regimes of radars on UAVs. Nowadays, the UAVs become cheap enough to perform scaled applications. Radars are also mature products and cheap enough to afford. The computational and communication ability of UAVs are high enough to perform our proposed extended algorithms. The implementation cost is also low enough.

\subsection{Scalability Analysis}

The scalability of our proposed method is good. However, in our proposed architecture, we only considered a single radar to track UAVs. It weakens the continuous tracking ability and system reliability. To improve the system performance, we will develop a multi-UAV cooperative application to form an ad hoc network. The vehicle radars can also connect to the UAV networks to make a supplement. The system communication ability can be guaranteed with the help of LTE, 5G, Wi-Fi and other advanced communication technology. The proposed method has potential in large-scale scenarios.

\section{Conclusions}

In this article, a special situation of tracking UAVs with mobile radars is discussed. We found that adopting EKF, UKF, and UCMKF directly in the ENU coordinates will cause filter-performance degradation or even divergence with mobile radars by theoretical motivation. That will degrade tracking performance of tracking UAVs with mobile radars, especially when radars move quickly. A new method is proposed to perform the filtering operation in the ECEF coordinates. The theory analysis and simulation results show that the proposed method can unify the motion model and guarantee the accuracy of the filtering process. With low cost and good scalability, it can constitute a UAV network in the future to improve the system performance. It is helpful to avert filtering performance degradation, and even divergence, when tracking UAVs with mobile radars. In the future, more motion models such as the constant-turn model, constant-acceleration model, and variable-acceleration models will 
be focused on to make it more reliable and practical when tracking UAVs. It is also significant in engineering applications.

Author Contributions: Conceptualization, Y.W.; Data curation, Y.W.; Methodology, Y.W., T.H. and M.K.; Software, T.H.; Supervision, T.H.; Validation, Y.W.; Writing - original draft, Y.W.; Writing - review \& editing, T.H. and M.K. All authors have read and agreed to the published version of the manuscript.

Funding: This research was funded by National Natural Science Foundation of China, grant number 61827901.

Acknowledgments: Partial results of this work have been published in IIKI 2018. The authors would like to thank the helpful comments of the anonymous reviewers.

Conflicts of Interest: The authors declare no conflict of interest.

\section{Appendix A}

In this part, we present some well- known equations to improve the readability of our paper.

$$
\begin{gathered}
h[X(k)]=\left[\begin{array}{c}
\sqrt{x^{2}+y^{2}+z^{2}} \\
a \tan \frac{y}{x} \\
a \sin \frac{z}{\sqrt{x^{2}+y^{2}}}
\end{array}\right] \\
H=\left.\frac{\partial h[X(k)]}{\partial X(k)}\right|_{X_{(k \mid k-1)}}=\left[\begin{array}{cccccc}
\frac{x}{r} & 0 & \frac{y}{r} & 0 & \frac{z}{r} & 0 \\
-\frac{y}{r_{1}^{2}} & 0 & \frac{z}{r_{1}^{2}} & 0 & 0 & 0 \\
-\frac{x z}{r_{1} r^{2}} & 0 & -\frac{y z}{r_{1} r^{2}} & 0 & \frac{r_{1}}{r^{2}} & 0
\end{array}\right]
\end{gathered}
$$

The calculation of the $\sigma$ sampling of the measurement vector are as follows.

1. Calculating the $\sigma$ sampling $\xi^{i}(k+1 \mid k), i=0,1, \ldots, 2 n$ after state transition equation:

$$
\left\{\begin{array}{l}
\xi^{i}(k+1 \mid k)=f\left(\xi^{i}(k \mid k)\right), i=0,1, \ldots, 2 n \\
X(k+1 \mid k)=\sum_{i=0}^{2 n} \omega_{i}^{m} \xi^{i}(k+1 \mid k) \\
P(k+1 \mid k)=\sum_{i=0}^{2 n} \omega_{i}^{c}\left[\xi^{i}(k+1 \mid k)-X(k+1 \mid k)\right]\left[\xi^{i}(k+1 \mid k)-X(k+1 \mid k)\right]^{T}+Q(k)
\end{array}\right.
$$

where

$$
\left\{\begin{array}{l}
\omega_{0}^{m}=\lambda /(n+\lambda) \\
\omega_{0}^{c}=\lambda /(n+\lambda)+\left(1-\alpha^{2}+\beta\right)
\end{array}, \omega_{i}^{m}=\omega_{i}^{c}=0.5 /(n+\lambda), i=1,2, \ldots, 2 n\right.
$$

In the above equation, $\beta$ describes the distribution information of $X$; the optimal value is $2 . \omega_{i}^{m}, \omega_{i}^{c}$ are weight coefficients of first- and second-order statistical properties, respectively.

2. Calculating the sampling of predicted value:

$$
\left\{\begin{array}{l}
\xi^{0}(k+1)=X(k+1 \mid k) \\
\xi^{i}(k+1)=X(k+1 \mid k)+(\sqrt{(n+\lambda) P(k \mid k)})_{i}, i=1, \ldots, n \\
\xi^{i}(k+1)=X(k+1 \mid k)-(\sqrt{(n+\lambda) P(k \mid k)})_{i}, i=n+1, \ldots, 2 n
\end{array}\right.
$$


3. Calculating the one-step prediction:

$$
\left\{\begin{array}{l}
\zeta^{i}(k+1 \mid k)=h\left[\xi^{i}(k+1)\right], i=0,1, \ldots, 2 n \\
Z(k+1 \mid k)=\sum_{i=0}^{2 n} \omega_{i}^{m} \zeta^{i}(k+1 \mid k) \\
P_{Z}(k+1)=\sum_{i=0}^{2 n} \omega_{i}^{c}\left[\zeta^{i}(k+1 \mid k)-Z(k+1 \mid k)\right]\left[\zeta^{i}(k+1 \mid k)-Z(k+1 \mid k)\right]^{T}+R(k) \\
P_{X Z}(k+1)=\sum_{i=0}^{2 n} \omega_{i}^{c}\left[\zeta^{i}(k+1 \mid k)-X(k+1 \mid k)\right]\left[\zeta^{i}(k+1 \mid k)-Z(k+1 \mid k)\right]^{T}
\end{array}\right.
$$

4. Filtering updates after fetching new measurement value:

$$
\left\{\begin{array}{l}
X(k+1 \mid k+1)=X(k+1 \mid k)+K(k+1)[Z(k+1)-Z(k+1 \mid k)] \\
K(k+1)=P_{X Z}(k+1) P_{Z}(k+1)^{-1} \\
P(k+1 \mid k+1)=P(k+1 \mid k)-K(k+1) P_{Z}(k+1) K(k+1)^{T}
\end{array}\right.
$$

The elements of matrix $R^{u}$ are given by:

$$
\begin{aligned}
& R_{x x}=-\lambda_{\theta}^{2} \lambda_{\eta}^{2} \widetilde{r}^{2} \cos ^{2} \widetilde{\theta} \cos ^{2} \widetilde{\eta}+\frac{1}{4}\left(\widetilde{r}^{2}+\sigma_{r}^{2}\right)\left(1+\lambda_{\theta}^{\prime} \cos 2 \widetilde{\theta}\right)\left(1+\lambda_{\eta}^{\prime} \cos 2 \widetilde{\eta}\right) \\
& R_{y y}=-\lambda_{\theta}^{2} \lambda_{\eta}^{2} \widetilde{r}^{2} \sin ^{2} \widetilde{\theta} \cos ^{2} \widetilde{\eta}+\frac{1}{4}\left(\vec{r}^{2}+\sigma_{r}^{2}\right)\left(1-\lambda_{\theta}^{\prime} \cos 2 \widetilde{\theta}\right)\left(1+\lambda_{\eta}^{\prime} \cos 2 \widetilde{\eta}\right) \\
& R_{z z}=-\lambda_{\eta}^{2} \widetilde{r}^{2} \sin ^{2} \widetilde{\eta}+\frac{1}{2}\left(\vec{r}^{2}+\sigma_{r}^{2}\right)\left(1-\lambda_{\eta}^{\prime} \cos 2 \widetilde{\eta}\right) \\
& R_{x y}=-\lambda_{\theta}^{2} \lambda_{\eta}^{2} \widetilde{r}^{2} \sin \widetilde{\theta} \cos \widetilde{\theta} \cos ^{2} \widetilde{\eta}+\frac{1}{4}\left(\vec{r}^{2}+\sigma_{r}^{2}\right) \lambda_{\theta}^{\prime} \sin 2 \widetilde{\theta}\left(1+\lambda_{\eta}^{\prime} \cos 2 \widetilde{\eta}\right) \\
& \left.R_{x z}=-\lambda_{\theta} \lambda_{\eta}^{2} \widetilde{r}^{2} \cos \widetilde{\theta} \sin \widetilde{\eta} \cos \widetilde{\eta}+\frac{1}{2} \widetilde{r}^{2}+\sigma_{r}^{2}\right) \lambda_{\theta}^{\prime} \lambda_{\eta}^{\prime} \cos \widetilde{\theta} \sin 2 \widetilde{\eta} \\
& R_{y z}=-\lambda_{\theta} \lambda_{\eta}^{2} \widetilde{r}^{2} \sin \widetilde{\theta} \sin \widetilde{\eta} \cos \widetilde{\eta}+\frac{1}{2}\left(\widetilde{r}^{2}+\sigma_{r}^{2}\right) \lambda_{\theta}^{\prime} \lambda_{\eta}^{\prime} \sin \widetilde{\theta} \sin 2 \widetilde{\eta} \\
& \lambda_{\theta}=e^{-\sigma_{\theta}^{2} / 2}, \lambda_{\theta}^{\prime}=\lambda_{\theta}^{4}, \lambda_{\eta}=e^{-\sigma_{\eta}^{2} / 2}, \lambda_{\eta}^{\prime}=\lambda_{\eta}^{4}
\end{aligned}
$$

\section{References}

1. Fragkos, G.; Tsiropoulou, E.E.; Papavassiliou, S. Disaster Management and Information Transmission Decision-making in Public Safety Systems. In Proceedings of the 2019 IEEE Global Communications Conference (GLOBECOM), Waikoloa, HI, USA, 9-13 December 2019; pp. 1-6.

2. Jiang, Z.J.; Cheng, X.G.; Peng, Y.Q. Research on UAV identification algorithm based on deeplearning. App. Elec. Tech. 2017, 43, 84-87.

3. Zhang, D.X. Research on electromagnetic interference mechanism of main remote control data link of UAV. J. Microwave 2016, 32, 90-96.

4. Zhao, J.C.; Fu, X.R.; Yang, Z.K.; Xu, F.T. Radar-Assisted UAV Detection and Identification Based on 5G in the Internet of Things. Wirel. Commun. Mob. Comput. 2019, 2019, 1-12. [CrossRef]

5. Shakhatreh, H.; Sawalmeh, A.H.; Al-Fuqaha, A.; Dou, Z.C.; Almaita, E.; Khalil, I.; Othman, N.S.; Khreishah, A.; Guizani, M. Unmanned Aerial Vehicles (UAVs): A Survey on Civil Applications and Key Research Challenges. IEEE Access 2019, 7, 48572-48634. [CrossRef]

6. Solomitckii, D.; Gapeyenko, M.; Semkin, V.; Andreev, S.; Koucheryavy, Y. Technologies for efficient amateur drone detection in 5G millimeter-wave cellular infrastructure. IEEE Commun. Mag. 2018, 56, 43-50. [CrossRef]

7. Schreiber, E.; Heinzel, A.; Peichl, M.; Engel, M.; Wiesbeck, W. Advanced buried object detection by multichannel, UAV/drone carried synthetic aperture radar. In Proceedings of the 2019 13th European Conference on Antennas and Propagation (EuCAP), Krakow, Poland, 31 March-5 April 2019; pp. 1-5.

8. Dill, S.; Schreiber, E.; Engel, M.; Heinzel, A.; Peichl, M. A drone carried multichannel Synthetic Aperture Radar for advanced buried object detection. In Proceedings of the 2019 IEEE Radar Conference (RadarConf), Boston, MA, USA, 22-26 April 2019; pp. 1-6. 
9. Zhu, L.Z.; Zhao, H.C.; Xu, H.L.; Lu, X.Y.; Chen, S.; Zhang, S.N. Classification of Ground Vehicles Based on Micro-Doppler Effect and Singular Value Decomposition. In Proceedings of the 2019 IEEE Radar Conference (RadarConf), Boston, MA, USA, 22-26 April 2019; pp. 1-6.

10. Harvey, A.C. Forecasting, Structural Time Series Models and the Kalman Filter; Cambridge University Press: Cambridge, UK, 1990.

11. Zekavat, R.; Buehrer, R.M. Handbook of Position Location: Theory, Practice and Advances; John Wiley \& Sons: Hoboken, NJ, USA, 2011.

12. Zhang, X.C.; Guo, C.J. Cubature Kalman filters: Derivation and extension. Chin. Phys. B. 2013, $22,128401$. [CrossRef]

13. Liu, Z.X.; Xie, W.X.; Wang, P. Tracking a target using a cubature Kalman filter versus unbiased converted measurements. In Proceedings of the 2012 IEEE 11th International Conference on Signal Processing, Beijing, China, 21-25 October 2012; pp. 2130-2133.

14. Bordonaro, S.; Willett, P.; Bar-Shalom, Y. Decorrelated unbiased converted measurement Kalman filter. IEEE Trans. Aerosp. Electron. Syst. 2014, 50, 1431-1444. [CrossRef]

15. Chen, V.C.; Li, F.; Ho, S.S.; Wechsler, H. Micro-Doppler effect in radar: Phenomenon, model, and simulation study. IEEE Trans. Aerosp. Electron. Syst. 2006, 42, 2-21. [CrossRef]

16. Basso, G.F.; De Amorim, T.G.S.; Brito, A.V.; Nascimento, T.P. Kalman Filter with Dynamical Setting of Optimal Process Noise Covariance. IEEE Access 2017, 5, 8385-8393. [CrossRef]

17. Roth, M.; Hendeby, G.; Gustafsson, F. EKF/UKF maneuvering target tracking using coordinated turn models with polar/Cartesian velocity. In Proceedings of the 17th International Conference on Information Fusion (FUSION), Salamanca, Spain, 7-10 July 2014; pp. 1-8.

18. Wu, W.; Jiang, J.; Feng, X.; Qin, X. A sequential converted measurement kalman filter with doppler measurements in ECEF coordinate system. Chin. J. Electron. 2016, 25, 139-145. [CrossRef]

19. Li, X.R.; Jilkov, V.P. A Survey of Maneuvering Target Tracking-Part III: Measurement Models. In Proceedings of the SPIE Conference on Signal and Data Processing of Small Targets, San Diego, CA, USA, 26 November 2001; pp. 423-446.

20. Elnashar, A.; El-saidny, M.A. LTE and LTE-A Overview. In Practical Guide to LTE-A, VoLTE and IoT: Paving the Way Towards 5G; John Wiley \& Sons: Hoboken, NJ, USA, 2018.

21. Vaughan-Nichols, S.J. Mobile WiMAX: The next wireless battle ground. Computer 2008, 41, 16-18. [CrossRef]

22. Rong, B.; Qian, Y.; Lu, K.; Chen, H.H.; Guizani, M. Call admission control optimization in WiMAX networks. IEEE Trans. Veh. Technol. 2008, 57, 2509-2522. [CrossRef]

23. Sun, S.; Kadoch, M.; Gong, L.; Rong, B. Integrating network function virtualization with SDR and SDN for 4G/5G networks. IEEE Netw. 2015, 29, 54-59. [CrossRef]

24. Qi, F.; Zhu, X.; Mang, G.; Kadoch, M.; Li, W. UAV network and IoT in the sky for future smart cities. IEEE Netw. 2019, 33, 96-101. [CrossRef]

25. Wang, L.; Che, Y.L.; Long, J.; Duan, L.; Wu, K. Multiple Access MmWave Design for UAV-Aided 5G Communications. IEEE Wirel. Commun. 2019, 26, 64-71. [CrossRef]

26. Zhang, L.; Zhao, H.; Hou, S.; Zhao, Z.; Xu, H.; Wu, X.; Wu, Q.; Zhang, R. A Survey on 5G Millimeter Wave Communications for UAV-Assisted Wireless Networks. IEEE Access 2019, 7, 117460-117504. [CrossRef]

27. Wu, Y.; Rong, B.; Salehian, K.; Gagnon, G. Cloud Transmission: A New Spectrum-Reuse Friendly Digital Terrestrial Broadcasting Transmission System. IEEE Trans. Broadcast. 2012, 58, 329-337. [CrossRef]

28. Wang, Y.; Phelps, T.; Kibaroglu, K.; Sayginer, M.; Ma, Q.; Rebeiz, G.M. 28 GHz 5G-Based Phased-Arrays for UAV Detection and Automotive Traffic-Monitoring Radars. In Proceedings of the 2018 IEEE/MTT-S International Microwave Symposium-IMS, Philadelphia, PA, USA, 10-15 June 2018; pp. 895-898.

29. Chan, E.C.L.; Baciu, G. Introduction to Wireless Localization: With IPhone SDK Examples; John Wiley \& Sons: Hoboken, NJ, USA, 2012.

30. Xu, G.C.; Xu, Y. GPS: Theory, Algorithms and Applications, 3rd ed.; Springer: Berlin/Heidelberg, Germany, 2016.

31. Julier, S.J.; Uhlmann, J.K. A New Extension of the Kalman Filter to Nonlinear Systems. In Proceedings of the Signal processing, sensor fusion, and target recognition VI, Orlando, FL, USA, 28 July 1997; pp. 182-193. 
32. Decker, B.L. World geodetic system 1984. In Proceedings of the Fourth International Geodetic Symposium on Satellite Positioning, Austin, TX, USA, 28 April-2 May 1986; pp. 69-92.

33. Mo, L.B.; Song, X.Q.; Zhou, Y.Y.; Sun, Z.K.; Bar-Shalom, Y. Unbiased converted measurements for tracking. IEEE Trans. Aerosp. Electron. Syst. 1998, 34, 1023-1027.

(C) 2020 by the authors. Licensee MDPI, Basel, Switzerland. This article is an open access article distributed under the terms and conditions of the Creative Commons Attribution (CC BY) license (http://creativecommons.org/licenses/by/4.0/). 